\title{
Von der Arbeit 4.0 zum Sinn 4.0? Über das Sinnerleben in der Arbeit in Zeiten der Digitalisierung
}

\author{
Friedericke Hardering
}

Online publiziert: 26. November 2020

(C) Der/die Autor(en) 2020

Zusammenfassung Die digitale Transformation verändert die Arbeitsgestaltung sowie das Arbeitserleben von Beschäftigten substanziell mit weitreichenden Konsequenzen für das gesellschaftliche Verständnis von Arbeit, aber auch für die Arbeitsansprüche von Beschäftigten. Vielfach werden in der aktuellen Debatte über die Digitalisierung Folgen für den Sinn von Arbeit oder das Sinnerleben von Beschäftigten angesprochen, ohne dass sich ein eindeutiges Bild ableiten lässt. Der Beitrag zielt darauf, die Auswirkungen der digitalen Transformation auf den Sinn von Arbeit wie auch auf das Erleben von Sinn im Bereich der Arbeit auszuloten. Dazu wird eine Heuristik genutzt, die verschiedene Diskursstränge über Sinn und Arbeit differenziert und den Stand der unterschiedlichen Debatten rekonstruiert. Der Beitrag zeigt die Unterschiedlichkeit der Debatten und die heterogenen Vertiefungsgrade der Diskussionen. Weiterhin kann gezeigt werden, dass weniger die digitale Arbeit bzw. Effekte der Digitalisierung selbst, sondern vielmehr die Veränderungen von Handlungsspielräumen Konsequenzen für das Sinnerleben haben, welche auf die betriebliche Leistungspolitik zurückzuführen sind.

Schlüsselwörter Digitalisierung · Digitale Arbeit · Aneignung von Arbeit · Sinnerleben in der Arbeit - Sinnvolle Arbeit - Arbeitssoziologie

F. Hardering $(\bowtie)$

FH Münster, Hüfferstraße 27, 48149 Münster, Deutschland

E-Mail: f.hardering@fh-muenster.de 


\title{
From work 4.0 to meaning 4.0? On meaning at work in times of digital transformation
}

\begin{abstract}
The digital transformation substantially changes the way work is organized and the work experience of employees with far-reaching consequences for the social understanding of work, but also for the work demands of employees. In the current debate on digitization, the consequences for the meaning of work or the experience of meaningfulness at work for employees are often addressed without a clear picture being derived. This contribution aims to explore the effects of digital transformation on the meaning of work as well as on the experience of meaningfulness in the field of work. To this end, a heuristic is used to differentiate different strands of discourse about meaning and work and to reconstruct the state of the various debates. The contribution shows the diversity of the debates and the heterogeneous degrees of depth of the discussions. Furthermore, it can be shown that it is not so much digital work or the effects of digitization itself, but rather the changes in the scope of action that have consequences for the experience of meaningfulness at work, which can be traced back to company performance policies.
\end{abstract}

Keywords Digitalization · Digital labour · Appropriation of work ·

Meaningfulness at work $\cdot$ Meaningful work $\cdot$ Sociology of work

\section{Einleitung}

Wer aktuell den Wandel der Arbeit nachzuvollziehen sucht, kommt an Thesen, Studien, Szenarien und Handlungsempfehlungen zur Digitalisierung der Arbeit kaum vorbei. Die Untersuchung der digitalen Transformation der Arbeit hat sich binnen weniger Jahre unter den Stichworten „Arbeit 4.0“ oder „Industrie 4.0“ zu einem zentralen Verhandlungsfeld arbeitssoziologischer Forschung entwickelt (Pfeiffer 2015; Hirsch-Kreinsen 2018; Jürgens et al. 2017). Das Interesse richtet sich dabei auf ganz unterschiedliche Aspekte wie die digitale Überwachung in der Logistik, die Chancen und Gefahren ortsungebundener Online-Plattformarbeit, die Einführung von Cyber-Physischen Systemen in der Industrie oder die problematischen oder positiven Aspekte von Social-Media-Nutzung am Arbeitsplatz (Carstensen 2017; Pongratz und Bormann 2017; Staab und Nachtwey 2016). Viele der Studien zielen darauf zu verstehen, welche Veränderungen sich für die Beschäftigung und Berufe, die Arbeitsorganisation, die Mensch-Technik-Interaktion, das Arbeitserleben, die Vereinbarkeit von Arbeit und anderen Lebensbereichen und die Interessensvertretung als Folge der Transformation ergeben, womit die Debatte in vielfältiger Weise an ältere Diskurse über neue Technologien und den Wandel von Arbeit anschließt (Heßler 2016).

Im Kontext der Auseinandersetzungen über die digitale Transformation wird intensiv über die Zukunft der Arbeit und auch über den Wandel des Sinns von Arbeit und des Sinnerlebens wie auch der Entfremdung in der Arbeit diskutiert. Aufgegriffen wird dieser Gedanke bei Rosa (2016, 2012, 2011), der die Digitalisierung als einen Treiber von Beschleunigungsprozessen identifiziert, die in der Arbeitswelt 
zu schwachen bzw. fehlenden Resonanzbeziehungen führen und damit Gefühle der Entfremdung forcieren können. Auch in Überlegungen zu einem digitalen Taylorismus, die davon ausgehen, dass durch neue digitale Technologien tayloristische Kontrollprinzipien radikaler umgesetzt werden (Nachtwey und Staab 2015), wird neben der Ausweitung der Kontrolle auch der mögliche Sinnverlust und die Entfremdung durch Standardisierung und Fragmentierung diskutiert. Gerade die Arbeit von Clickworkern, die sich dadurch auszeichnet, dass auf digitalen Plattformen eine sehr begrenzte Aufgabe online bearbeitet wird, kann als Inbegriff neuer entfremdeter Arbeit gesehen werden: Die Clickworker arbeiten, ohne den Kontext der Tätigkeit im Gesamtgefüge zu sehen, sie sind abhängig von der Online-Plattform, über die die Aufträge vermittelt werden, und erhalten einen sehr geringen Lohn (Graham et al. 2017).

Jenseits der fachwissenschaftlichen Debatte wird die Frage, was die Digitalisierung mit dem Sinn der Arbeit macht, medial breit aufgegriffen. Vielfach wird die Digitalisierung als Startpunkt betrachtet, um über den Sinn der Arbeit neu nachzudenken. So beschreibt das Zukunftsinstitut den Megatrend New Work folgendermaßen: „Wenn Maschinen künftig bestimmte Arbeiten besser verrichten können als der Mensch, beginnen wir, über den Sinn der Arbeit nachzudenken. Wenn die Arbeit uns nicht mehr braucht, wofür brauchen wir dann die Arbeit? New Work beschreibt einen epochalen Umbruch, der mit der Sinnfrage beginnt und die Arbeitswelt von Grund auf umformt." ${ }^{1}$ Gefragt wird in solchen Beiträgen nach dem Potenzial für ein neues Arbeitsverständnis, wenn smarte Maschinen die Arbeitenden ersetzen. In einem Artikel der Zeit (Broy und Precht 2017) wird die Frage aufgeworfen: „Macht die Digitalisierung die Welt nicht arm und leer - leer an Sinn, Arbeit, Erfahrung und Gefühl?" Entsprechend dieser Sorge wird über die Gefahren von neuen Entfremdungspotenzialen durch digitale Arbeit nachgedacht, wenn die Autonomie der Beschäftigten durch das Diktat der Maschinen verlorengeht, die Arbeit noch stärker fragmentiert wird und man sich durch neue Technologien sozial isoliert fühlt (Vašek 2017). Neben pessimistischen Einschätzungen finden sich aber auch optimistischere Diagnosen, die Chancen für das Sinnerleben in der Arbeit und auch für eine Neubestimmung des Arbeitsbegriffes benennen. So wird eine Chance für das Arbeitserleben von Beschäftigten gesehen, wenn durch die Digitalisierung als sinnlos erlebte Routinetätigkeiten wegfallen (Badura 2018). Damit würde die Qualität der Arbeit erhöht und Beschäftigte würden verstärkt in Bereichen eingesetzt, in denen die Arbeit sich durch eine höhere Aufgabenkomplexität auszeichnet.

So eindrücklich die Thesen über neue Chancen oder Probleme für das Erleben von Sinn in der Arbeitswelt durch die Digitalisierung sind, ist die Debatte charakterisiert durch eine Unübersichtlichkeit der Argumente. Anhand der obigen Beispiele wird zudem deutlich, dass sich die Diskussion auf unterschiedlichen Ebenen bewegt: So stellt sich die Sinnfrage in den vorgenannten Beispielen sowohl auf der Ebene der Arbeitserfahrung, also der Erfahrung von Sinn oder der Entfremdung, oder auf der Ebene der allgemeinen Bedeutung und Wichtigkeit von Arbeit in Zeiten sich wandelnder Technologien. Durch die Heterogenität der Diskussionsführung bleiben somit wesentliche Fragen offen: Was ist bisher über das Erleben von Sinn

\footnotetext{
1 https://www.zukunftsinstitut.de/dossier/megatrend-new-work/. Zugegriffen: 16.10.2020.
} 
in der digitalen Arbeit bekannt? Welche positiven und negativen Effekte für das Sinnerleben zeichnen sich in unterschiedlichen Bereichen der Arbeit ab? Und welche Dynamiken offenbaren sich mit Blick auf die Bedeutung von Arbeit?

Der Beitrag knüpft an diese Fragen an und zielt darauf, aktuelle Studien und Befunde zum Erleben von Sinn in Zeiten der Digitalisierung einzuordnen und zu systematisieren, um auf dieser Grundlage bisherige Erkenntnisse und weitere Forschungsbedarfe abzuleiten. Dazu wird eine Heuristik genutzt, die verschiedene Ebenen der Diskussion über den Sinn in der Arbeitswelt differenziert. Auf dieser Basis zeigt sich, dass sowohl der Kenntnisstand wie auch der Vertiefungsgrad in den unterschiedlichen Diskussionsfeldern deutlich voneinander abweichen. Während es sich bei der Diskussion um den gesellschaftlichen Sinn der Arbeit um einen spekulativen Zukunftsdiskurs handelt, finden sich in einzelnen Studien über die Erforschung des subjektiven Sinnerlebens im Kontext digitaler Arbeit Hinweise auf mögliche Einflussfaktoren auf das Sinnerleben. Auf der Basis der wenigen Befunde zum Sinnerleben in Zeiten der Digitalisierung deutet sich an, dass die Folgen für das Sinnerleben durch die Digitalisierung sehr unterschiedlich ausfallen und die Rolle der Technik selbst weitaus weniger ins Gewicht fällt, als dies Steuerungs- und Machtwirkungen tun, die mit der Technologienutzung einhergehen.

Der Beitrag gliedert sich wie folgt: In einem ersten Schritt wird auf die aktuelle Digitalisierungsdiskussion sowie auf die Erforschung sinnvoller Arbeit eingegangen. Anschließend wird die Heuristik zur Differenzierung der Diskurse vorgestellt. Entlang der drei Ebenen werden die verschiedenen Diskurse über den gesellschaftlichen Sinn der Arbeit, den individuellen Sinn der Arbeit und das Erleben von Sinn in der Arbeit im Feld der Digitalisierung differenziert und es wird auf bisherige Befunde eingegangen. Ausführlicher werden die Erkenntnisse im Bereich des Sinns in der Arbeit vertieft, da sich hier einerseits Studien mit unmittelbarem Bezug zur Sinnforschung zeigen, andererseits aber auch Impulse aus der aktuellen Digitalisierungsforschung wie der Forschung zu Technikaneignung aufgenommen werden.

Der Gewinn eines solchen Durchganges zeigt sich vor allem in zwei Bereichen: Erstens wird mit der Systematisierung verschiedener Diskussionslinien eine Präzisierung der aktuellen Diskussion über Fragen des Sinns von Arbeit in Zeiten der Digitalisierung angeregt. Dadurch wird es möglich, die Debatte über veränderte gesellschaftliche Arbeitsverständnisse und deren Identifikationspotenzial vom subjektiven Sinnerleben der Beschäftigten zu trennen und so die unterschiedlichen Erkenntnisfortschritte der jeweiligen Debatten zu differenzieren.

Zweitens bietet sich für die aktuelle Erforschung der Digitalisierung die Chance, bisherige Blindstellen des Verständnisses der subjektiven Erfahrung von Digitalisierungsprozessen anhand der konkreten Erforschung des Sinnerlebens aufzuzeigen. Vielfach wurde angemerkt, dass das subjektive Erleben und die Wahrnehmung von Digitalisierungsprozessen noch unzureichend verstanden sind (Hirsch-Kreinsen 2018; Bundesministerium für Arbeit und Soziales 2016; DGB-Index Gute Arbeit 2017). Zwar gibt es Hinweise dafür, dass die Digitalisierung häufig mit einer Belastungszunahme assoziiert wird (Pfeiffer 2015; DGB-Index Gute Arbeit 2017). Wie aber genau Dynamiken von Belastungen und Ressourcen zu verstehen sind, ist bisher noch weitgehend offen. Mit Sinnerleben und Aneignung werden Konzepte angeführt, die ermöglichen, die subjektiven Arbeitserfahrungen der Beschäftigten 
im Kontext der Digitalisierung zu erschließen. Dadurch können bisherige Erkenntnisse über wahrgenommene Autonomie oder veränderte Subjektivierungsweisen im Kontext von Arbeit vertieft werden.

\section{Digitalisierung und Sinn (in) der Arbeit: Konzepte und Forschungsstand}

Die Digitalisierung der Arbeitswelt steht als Stichwort für ein breit gefächertes Untersuchungsfeld, welches sich im Kontext veränderter digitaler Technologien entwickelt und durch industriepolitische Weichenstellungen, Initiativen und Fördermaßnahmen rasch an Relevanz gewonnen hat (Briken et al. 2017; Hirsch-Kreinsen et al. 2018; Pfeiffer 2015). Trotz des enormen Interesses am Gegenstand sind zentrale Begrifflichkeiten der Debatte wie Digitalisierung, Arbeit 4.0 oder digitale Arbeit bisher uneindeutig geblieben (Hoose 2018). Auch wenn von der Digitalisierung gesprochen wird, deutet sich an, dass es die Digitalisierung als fest umrissenes Phänomen nicht gibt, vielmehr verbergen sich hinter dem Begriff ganz unterschiedliche Veränderungsdynamiken. Hirsch-Kreinsen (2018, S. 16) definiert Digitalisierung als „Prozess des sozio-ökonomischen Wandels [...] der durch Einführung digitaler Technologien, darauf aufbauender Anwendungssysteme und vor allem ihrer Vernetzung angestoßen wird.“ Die heterogenen Folgen dieser Transformation für die Erwerbsarbeit, aber auch für andere Tätigkeitsformen sowie für das Verständnis davon, was Arbeit ist und sein soll, bilden den Kern der Debatte über den Prozess der Digitalisierung der Arbeitswelt. Die Digitalisierung umfasst damit weit mehr als das Verständnis von Arbeit mit digitalen Technologien, also dem, was gemeinhin als digitale Arbeit verstanden wird (Hoose 2018). Digitale Arbeit bezeichnet nach Schwemmle und Wedde (2012) üblicherweise Tätigkeiten, die mit digitalen Arbeitsmitteln an digitalen Objekten erfolgen. Orlikowski (2016) hingegen argumentiert, dass Arbeit heutzutage fundamental mit dem Digitalen verstrickt ist, selbst wenn die eigentliche Tätigkeit keinen Umgang mit digitalen Geräten beinhaltet. Als Beispiel führen sie die Arbeit von Reinigungskräften in Hotels an, die an sich nicht digital ist, gleichwohl aber durch Bewertungsprozesse über Internetportale zu einem Gegenstand der Bewertung in der digitalen Welt wird. Es gibt also nicht die digitale Arbeit, vielmehr finden sich vielfältige Formen derselben und es bietet sich an, engere und weitere Verständnisse digitaler Arbeit auszudifferenzieren (Hoose 2018). Im Beitrag wird einerseits die Digitalisierung der Arbeit als umfassendere Perspektive auf den sich vollziehenden sozio-technischen Wandel betrachtet, andererseits werden in den Studien unterschiedliche Formen digitaler Arbeit in den Blick genommen, die im Umfang der Nutzung digitaler Informations- und Kommunikationstechnologien voneinander abweichen.

Wie wird nun aktuell über den Sinn und die Digitalisierung diskutiert? Um die heterogenen Zweige der Debatte zu differenzieren, wird zunächst auf die Erforschung sinnvoller Arbeit eingegangen, um auf dieser Grundlage verschiedene Diskussionsebenen zu identifizieren. Sowohl im deutschsprachigen wie internationalen Raum lässt sich in den letzten Jahren ein Aufschwung der Erforschung von Sinn in der Arbeit beobachten (Bailey et al. 2018; Badura et al. 2018; Hardering 2015; Jürgens 
2014; Nies 2015; Voswinkel 2015). Die Ursachen für die verstärkte Thematisierung sind vielfältig und liegen auf der einen Seite in substanziellen Veränderungen der Arbeitswelt durch die Digitalisierung, die Globalisierung, aber auch den demografischen Wandel, die allesamt das Verständnis von Arbeit irritieren. ${ }^{2}$ Auf der anderen Seite sind neben diesen Entwicklungen aber auch Fortschritte der wissenschaftlichen Diskussion über meaningful work zu nennen, die zu einer intensiveren Wissensproduktion beigetragen haben. Trotz einer intensiveren Diskussion über die Bedeutung von meaningful work sind viele der begrifflichen Probleme noch die gleichen wie vor einigen Jahren: Der Sinnbegriff ist nach wie vor unscharf und konsistente Definitionen Mangelware, wie vielfach kritisiert wird (Bailey et al. 2018; Jürgens 2014; Hardering 2015). ${ }^{3}$ Die Unschärfe hat dazu beigetragen, dass mittlerweile zahlreiche Bemühungen der Systematisierung vorliegen (Bailey et al. 2018).

\section{Drei Diskurse über Digitalisierung und Sinn}

Als fruchtbar hat sich die Unterscheidung zwischen dem Sinn der Arbeit und dem Sinn in der Arbeit herauskristallisiert (Hardering 2015; Rosso et al. 2010; Schnell et al. 2013), die zunächst grundlegend zwischen der Bedeutung von Arbeit und der subjektiv erlebten Sinnhaftigkeit von Arbeit unterscheidet. Weiterhin kann die Bedeutung von Arbeit ausdifferenziert werden in die gesellschaftliche Bedeutung von Arbeit und die individuelle Bedeutung von Arbeit. ${ }^{4}$ Diese Diskussionsebenen des gesellschaftlichen und individuellen Sinns der Arbeit sowie des Sinns in der Arbeit ermöglichen, die Diskussion über Sinn im Kontext der Digitalisierungsdebatte zu strukturieren und den Vertiefungsgrad der jeweiligen Diskussion nachzuvollziehen.

\subsection{Warum arbeiten wir? Die Debatte über den gesellschaftlichen Sinn der Arbeit}

Über den gesellschaftlichen Sinn und damit über die Frage, was Arbeit ist und sein soll, welchen Stellenwert Erwerbsarbeit neben anderen Tätigkeitsformen und neben Nichtarbeit für die Gesellschaft hat und wie die aktuelle Erwerbsarbeitszentrierung aufgebrochen werden kann, wird aktuell in Dialogprozessen rund um das Thema Arbeit 4.0., aber auch in der aktuellen Debatte über erweiterte Arbeitskonzepte (Littig und Spitzer 2011; Nierling und Krings 2019) und insbesondere über das bedingungslose Grundeinkommen verhandelt. Letztere Diskussion hat durch die Sorge über einen massenhaften Arbeitsplatzverlust neuen Schwung und neue Befürwortende bekommen (Bregman 2019; Vobruba 2019; Precht 2018). Das Grundeinkommen

\footnotetext{
2 Vgl. hierzu die Diskussion über veränderte Arbeitsansprüche nachrückender Generationen, beispielsweise der Generation Y (Hardering 2018).

3 Die unterschiedlichen Verständnisse von Sinn im Kontext von Arbeit zeigen sich auch im Fehlzeitenreport 2018, der sich explizit mit dem Thema befasst und in dem ganz unterschiedliche Sinn-Verständnisse genutzt werden. Eine Systematisierung des Sinn-Begriffs oder zumindest ein Verweis auf die heterogenen Verständnisse fehlt (Badura et al. 2018).

4 Zur ausführlichen Differenzierung siehe Hardering (2020).
} 
bildet nur einen Bezugspunkt dieser Diskussion, die u. a. auch durch die Post-WorkBewegung $^{5}$ angefacht wird (Rosswog 2018; Mason 2016; Srnicek und Williams 2016). Ein gemeinsamer Bezugspunkt der sehr unterschiedlichen Denkströmungen ist die Annahme, dass Arbeit unter den Vorzeichen gegenwärtiger Veränderungen, die sich nicht ausschließlich durch die Digitalisierung erklären lassen, auch jenseits des fordistischen Normalarbeitsverhältnisses gedacht werden kann. Dies eröffnet die Möglichkeit, andere Formen des Arbeitens bzw. Tätigseins normativ aufzuwerten.

Die Digitalisierung wird in diesen Diskussionen unterschiedlich bewertet: Sie kann die Befreiung von Arbeit ermöglichen, wenn durch immer bessere Algorithmen und Automatisierungsmöglichkeiten Arbeitsprozesse nicht mehr von Menschen ausgeführt werden müssen. Oder die Digitalisierung kann dem Bedrohungsszenario entsprechend dafür sorgen, dass es zu einem massenhaften Arbeitsplatzverlust kommen wird. ${ }^{6}$ Grundlage des Bedrohnungsszenarios sind Studien über die Substituierbarkeitspotenziale wie die vielfach zitierte Studie von Frey und Osborne (2013). Unabhängig davon, wie optimistisch oder pessimistisch man die mögliche Befreiung von Arbeit bewertet, evoziert die Digitalisierung der Arbeitswelt ein neues Nachdenken darüber, wie sich die Arbeitswelt selbst definieren möchte, was heutzutage noch als Arbeit gilt und wie Arbeit menschengerecht gestaltet werden kann (Hardering 2017a). Vieles von dem, was aktuell zum Sinn der Arbeit oder zur Zukunft der Arbeit durch neue Technologien verhandelt wird, findet sich bereits in den älteren Diskussionen über die Zukunft bzw. das Ende der Arbeit wieder und schließt auch mit Blick auf die dominanten Narrationslinien an die in der Vergangenheit geführten Debatten an (Heßler 2016; Rifkin 1995; Matthes 1983). Auch die Polarisierung des Diskurses in einen Chancen- bzw. Gefahrendiskurs zeigt sich bereits in Vorgängerdiskursen (Heßler 2016). So zeigt Heßler (2016) anhand des Automatisierungsdiskurses, dass bereits in den früheren Phasen des Diskurses in den 1950er-, 1970erund 1980er-Jahren vonseiten der Unternehmen die Chancen auf Erhöhung der Wettbewerbsfähigkeit, Verbesserung der Arbeit und Erhöhung der Freizeit durch Automatisierungsprozesse in der Industrie herausgestrichen wurden, wohingegen seitens der Gewerkschaften oder der Soziologie eher Gefahren der Automatisierung angesprochen wurden. Gerade die Argumentationen um die Ersetzung des Menschen, das Verschwinden der Arbeit und das Ende der Arbeitsgesellschaft weisen, wie Heßler (2016) zeigt, eine deutliche Persistenz auf. Auch in der aktuellen Diskussion über Digitalisierung wird von einem veränderten Verständnis von Arbeit ausgegangen, doch ähnlich wie in den älteren Debatten verbleiben die Vermutungen darüber, welche Bedeutung Arbeit zukünftig beigemessen wird und was als Arbeit definiert wird, abstrakt. Gegenwärtig hat dies eher zu einer Öffnung der Diskussion denn zu einer Verengung auf neue konkrete Fluchtpunkte für ein zukunftsfähiges Arbeitskonzept beigetragen. Insgesamt erfährt somit die Frage nach dem gesellschaftlichen Sinn von Arbeit Auftrieb durch die Digitalisierungsdiskussion, substanzielle Veränderungen in Bezug auf das Selbstverständnis der Arbeitsgesellschaft zeigen sich hingegen bisher nicht.

\footnotetext{
5 Post Work steht als Konzept für unterschiedliche Ansätze, die die Erwerbsarbeitsfixierung von Arbeitsgesellschaften kritisieren und ein neues Arbeitsverständnis fordern.
}

6 Zur Einschätzung für Deutschland siehe Bonin et al. (2015). 


\subsection{Die Wichtigkeit von Arbeit für das Leben - Die Debatte über den individuellen Sinn der Arbeit}

Auch die Fragen nach der Bedeutung von Arbeit für das eigene Leben oder dem Warum der eigenen Arbeit gewinnen durch Digitalisierungsprozesse neue Brisanz. Welchen Stellenwert Arbeit im Leben haben soll, muss gerade durch die Möglichkeit des orts- und zeitunabhängigen Arbeitens immer wieder neu beantwortet werden. Mit mobilen digitalen Geräten und Informations- und Kommunikationstechnologien wie E-Mail, Cloudarbeit oder Videotelefonie besteht für einige Beschäftigtengruppen die Möglichkeit für räumlich und zeitlich flexibles Arbeiten (Carstensen 2015). Durch diese Entgrenzung von Arbeit sind die Grenzziehungsleistungen von den Beschäftigten selbst zu erledigen. Für Beschäftigte gehen die neuen technischen Möglichkeiten so mit der Anforderung einer erhöhten Selbststeuerungsfähigkeit einher. Sie müssen eigenständig die Arbeitszeit von der Familien- bzw. Freizeit abgrenzen. Praktisch geht es um ein Mehr an Entscheidungen der Priorisierung, und diese Entscheidungen werfen auch immer Fragen nach der Wichtigkeit und Dringlichkeit der jeweiligen Aufgabe auf.

Zudem geht die Möglichkeit allgegenwärtiger Arbeitskommunikation via Smartphone oder Laptop häufig mit dem Gefühl einher, permanent zur Verfügung stehen zu müssen und nicht abschalten zu können. Dieses Gefühl kann dazu beitragen, dass das detachment, die mentale Abgrenzung von der Arbeit erschwert wird (Wendsche und Lohmann-Haislah 2017). Die gesundheitlichen Implikationen der individuellen Bedeutungszuschreibung von Arbeit sind vielschichtig: Eine positive Verbindung zur Arbeit und eine hohe individuelle Bedeutsamkeit gelten als Schutz gegen Belastungen; gleichzeitig kann gerade in belastenden Arbeitsumfeldern eine hohe subjektive Zentralität der Arbeit zu Gefühlen der Erschöpfung und zu psychischen Erkrankungen beitragen. ${ }^{7}$ Kontrovers wird diskutiert, wie genau die Verbindungslinien zwischen der Digitalisierung der Arbeitswelt, der Zunahme von Zeit- und Leistungsdruck, subjektiv erlebter Arbeitsüberforderung und der Entstehung psychischer Erkrankungen beschaffen ist (DGB-Index Gute Arbeit 2017; Gimpel et al. 2018; Rosa 2016). Bisherige Untersuchungen deuten darauf hin, dass die Digitalisierung mit einer Belastungszunahme in Verbindung steht (DGB-Index Gute Arbeit 2017).

Für die individuelle Wichtigkeit der Arbeit im Leben ist aber noch eine andere Tendenz interessant, die auch als Reaktion auf die Überforderungspotenziale der Arbeitswelt zu werten ist: Besonders in der Online-Arbeitswelt zeigt sich ein neues Nachdenken über und Ausprobieren von Arbeitsformen, deren Grundlage häufig eine Soloselbstständigkeit ist und die sich dadurch auszeichnet, dass über Onlinemarketing eigene Produkte verkauft werden, oder die eigene Arbeitskraft auf Crowdworking-Plattformen angeboten wird. Als attraktives Zielbild gilt hier u.a. das Leben als „,digitale Nomaden“, die unabhängig von ihrer Arbeit ihren Aufent-

\footnotetext{
7 Hierzu sind die Debatten über Arbeit, Digitalisierung und Gesundheit zu nennen (Kämpf 2015; MüllerThur et al. 2018; Ohlbrecht 2018; DGB-Index Gute Arbeit 2017). Darüber hinaus wird noch grundlegender über die Bedeutung des Wandels der Arbeit und der Beschaffenheit des modernen Kapitalismus für die Zunahme psychischer Erkrankungen diskutiert (Neckel und Wagner 2013; Dornes 2016).
} 
haltsort bestimmen und über diese Umkehrung auch das Verhältnis von Arbeit und Leben neu gewichten (Welskop-Deffaa 2018). So prekär diese Arbeitsformen sind, beinhalten sie neue Perspektiven und Praxisformen, wenn es um das Verhältnis von Arbeit und Leben geht (Pongratz und Bormann 2017). Solche Tendenzen lassen sich auch als Beitrag zum Überdenken des gesellschaftlichen Sinns von Arbeit begreifen.

\subsection{Die Arbeit als sinnvoll erleben - Der Diskurs über den Sinn in der Arbeit}

Wird der Sinn in der Arbeit verhandelt, ist eine Perspektive angesprochen, in der es um das subjektive Arbeitserleben, die Identifikation mit der Arbeit und das Wissen um die Nützlichkeit der Arbeit für einen selbst und für andere geht. Wer Sinn in der Arbeit erlebt, weiß, warum und wofür er oder sie arbeitet, empfindet eine Verbindung zu seiner Arbeit und erlebt eine Übereinstimmung von persönlichen Werten im Arbeitskontext (Hardering 2017b; Lips-Wiersma et al. 2016). Die meisten Klagen über neue Formen der Entfremdung wie auch über die neuen Belastungen, die im Zusammenspiel mit der Digitalisierung auftreten, verweisen auf das beeinträchtigte Erleben von Sinn in der Arbeit. Angenommen wird hier, dass durch neue Aufgabenzuschnitte infolge der Digitalisierung die Arbeit vereinfacht wird, an Aufgabenkomplexität verliert und in der Folge als sinnlos wahrgenommen wird. Doch neben der Fragmentierung der Arbeit sind auch weitere mögliche Hürden für das Sinnerleben zu nennen: hierzu zählen Einschnitte der Autonomie, die durch algorithmische Steuerung eintreten können, der fehlende persönliche Bezug zu Vorgesetzten oder Kolleg*innen durch digitale Steuerung und Kommunikation, der als belastend wahrgenommen werden kann, ein veränderter Bezug zu Arbeitsgeräten und Arbeitsergebnissen sowie ein Relevanzverlust der analogen Arbeitswelt, der sich dann einstellen kann, wenn sich die Tätigkeit fast ausschließlich im digitalen Raum verorten lässt.

Bisher sind es nur einzelne Studien, welche die Folgen der Digitalisierung im Allgemeinen oder Formen digitaler Arbeit aber konkret für die Aneignung von Arbeit und das Erleben von Sinnerfüllung (meaningfulness) bei der Arbeit untersuchen (Eisenmann und Wienzek 2018; Stein et al. 2018; Symon und Whiting 2019; Kost et al. 2018). Doch bieten auch Untersuchungen aufschlussreiche Erkenntnisse, die nicht unmittelbar Fragen des Sinnerlebens adressieren, sondern den weiteren Begriff der Aneignung von Arbeit bzw. von Technik nutzen. Daher sollen neben den wenigen Befunden mit direktem Bezug zur Meaningful-work-Forschung auch Befunde über Technikaneignung einbezogen werden.

\section{Sinn finden in digitaler Arbeit}

Befunde über das Sinnerleben in digitalen Arbeitskontexten stammen aus Untersuchungen über unterschiedliche Beschäftigtengruppen wie hochqualifizierten Akademikern oder Beschäftigten auf Microtasking-Plattformen. Weiterhin unterscheiden sich Art, Ausmaß und Beschaffenheit der digitalen Arbeit, sodass sich kein übergreifendes Bild zeichnen lässt. In der Studie von Stein et al. (2018) über datification work von Hochschulbeschäftigten wird der Einfluss der Datafizierung auf die Erfahrung 
von Sinnerfüllung (meaningfulness) untersucht. Unter datification work verstehen die Autor*innen das Einpflegen von Daten über die persönliche Arbeitsleistung in ein digitales Datenverarbeitungssystem. Stein et al. (2018) zeigen, dass datification work ambivalente Wirkungen auf das Sinnerleben haben kann: Sie kann Professionelle dabei unterstützen, Erfolge und Leistungen sichtbar zu machen und so helfen, den Wert der Arbeit zu verdeutlichen. Auch die Möglichkeit der Veränderung und Verbesserung des Datenverarbeitungssystems als aktive Form der Technikaneignung kann zu einem positiven Sinnerleben beitragen. Sofern das digitale Datenverarbeitungssystem starr ist und wenig Flexibilität bei der Eingabe ermöglicht, kann es negative Folgen für das Sinnerleben haben und mit Entfremdung einhergehen. Stein et al. (2018) resümieren, dass entgegen vieler Thesen, die in der Digitalisierung vor allem neue Hürden des Sinnerlebens sehen, es maßgeblich von der Gestaltung der Arbeit abhängig ist, ob sie zu Sinnerfüllung oder Sinnverlust beiträgt. Entscheidend sind somit nicht zuletzt die Gestaltungsspielräume, die den Beschäftigten eingeräumt werden, um sich die Technologien anzueignen. Wenngleich Stein et al. (2018) auch die subjektiven Gestaltungsleistungen der Arbeit in den Blick nehmen, liegt der Akzent der Untersuchung auf den Auswirkungen einer spezifischen Tätigkeitsform auf das Sinnerleben. Hinter dieser Perspektive steht eine Strömung der Meaningfulwork-Forschung, die Strukturen wie der Arbeitsgestaltung oder der Organisation eine entscheidende Funktion für das Sinnerleben beimisst (Bailey et al. 2018).

Die Studie von Kost et al. (2018) fragt, wie Microworker, die bei Amazon Mechanical Turk arbeiten, Sinn in ihrer Arbeit konstruieren. Die Autor*innen identifizieren vier Sinnquellen (sources of meaningfulness): Belohnungen, Selbstverbesserung, moralische und soziale Motive. Als Belohnung spielen Geld ebenso wie Autonomie eine entscheidende Rolle.

Die Studie zeigt auch, dass die Microworker ihre Arbeitsrollen durch verschiedene Praktiken wie Wissenserweiterung oder Geben durch Arbeit (um-)gestalten. Kost et al. (2018) folgen in der Anlage ihrer Untersuchung primär der humanistischen Strömung der Sinnforschung (Bailey et al. 2017), die die Gestaltungsleistungen und Zuschreibungsleistungen von Subjekten in den Fokus stellt und Sinn so als Ergebnis subjektiver Deutungsleistungen betrachtet. ${ }^{8}$ Diese Perspektive geht nicht zuletzt auf die Arbeiten von Viktor Frankl und Aaron Antonovsky zurück und wird in verschiedenen Studien wie beispielsweise der Untersuchung von Isaksen (2000) aufgegriffen.

Weiterhin ist die Studie von Eisenmann und Wienzek (2018) zu nennen, die anhand einer Diskussion von Entwicklungsszenarien digitaler Arbeit zeigt, dass die Digitalisierung sowohl Chancen als auch Gefahren für das Sinnerleben beinhaltet. Die Argumentation fußt allerdings auf einem weiten Verständnis des Sinnerlebens, welches eher auf die Bedeutung von Arbeit für das Leben und Aspekte allgemeiner Arbeitszufriedenheit abstellt als auf die Erfahrung von Sinnhaftigkeit im Arbeitskontext. Entsprechend werden allgemeine Bedeutungen und Funktionen von Arbeit für das Leben benannt wie materielle Bedürfnisse oder die Bedeutung von Erwerbsarbeit für die Identität. Somit ist der Beitrag nicht im engeren Sinne der Erforschung des

\footnotetext{
${ }^{8}$ Zudem findet sich auch die funktionalistische Perspektive, die allerdings in der Studie wesentlich auf den Einfluss der Arbeit auf andere bezogen wird.
} 
Sinnerlebens im Arbeitskontext zuzuordnen, sondern eher dem individuellen Sinn der Arbeit. Nichtsdestoweniger treffen Eisenmann und Wienzek auch Aussagen über das Sinnerleben, sodass sie an dieser Stelle Erwähnung finden. Die Autoren (2018) gehen davon aus, dass durch die Digitalisierung das Szenario einer Polarisierung und damit einer Spaltung der Arbeit in hochqualifizierte und geringqualifizierte Tätigkeiten bei einem gleichzeitigen Rückgang mittlerer Tätigkeitsniveaus wahrscheinlich wird. Damit steige den Autoren zufolge die Gefahr monotoner und damit auch gesundheitlich belastender Arbeit. Der Beitrag lässt sich durch die Akzentuierung der Qualifikationsniveaus einer Strömung zuordnen, die die Arbeitsgestaltung oder andere Faktoren wie das Qualifikationsniveau als bestimmenden Faktor des Sinnerlebens sieht.

Die wenigen Studien zeigen zunächst, dass sich auch in der Erforschung des Sinns im Kontext von Digitalisierung unterschiedliche Forschungsperspektiven ausfindig machen lassen, die auf je andere Strömungen der Meaningful-work-Forschung zurückzuführen sind. Ebenso zeigen die Studien, dass sich auch unter den Vorzeichen der Digitalisierung aus der Sinnforschung bekannte Mechanismen der Sinnzuschreibung sowie hemmende und fördernde Faktoren des Sinnerlebens finden lassen. Deutlich werden in allen beschriebenen Untersuchungen die Parallelen zu Befunden anderer Untersuchungen über das Sinnerleben, die sich nicht explizit mit der Digitalisierung beschäftigt haben: So akzentuiert die Studie von Stein et al. (2018) die Bedeutung der wahrgenommenen Handlungsspielräume für das Sinnerleben; ein Befund, der sich seit den Arbeiten von Hackman und Oldham (1980) als grundlegende Annahme der Sinnforschung beschreiben lässt. Weiterhin zeigen die Studien, dass das Selbst und das Andere für die Erfahrung von meaningfulness eine bedeutende Rolle spielen, was sich auch in anderen Studien zum Sinnerleben zeigt (LipsWiersma 2001; Hardering 2017b; Rosso et al. 2010).

Zusammengenommen zeigen die noch spärlichen Befunde über den Sinn in der Arbeit den Bedarf an weiteren Untersuchungen, die sich der konkreten Erfahrung des Sinnerlebens im Zusammenspiel mit digitaler Arbeit oder auch im weiteren Sinne mit Digitalisierungsprozessen widmen. Was sich in den Studien andeutet, ist, dass die Digitalisierung selbst kaum als eigenständiger Erfahrungsbereich in den Blick tritt; vielmehr sind es die ermächtigenden oder beschränkenden Wirkungen im Kontext organisationaler und gesellschaftlicher Machtverhältnisse, die auf struktureller Seite Einfluss auf das Sinnerleben nehmen. Zudem zeigt sich die Relevanz der Differenzierung unterschiedlicher Forschungsperspektiven, die entweder auf die strukturellen Voraussetzungen und Rahmenbedingungen fokussieren oder die individuellen Zuschreibungsleistungen von Sinn in den Fokus stellen.

In Ergänzung zu den Befunden, die unmittelbar das Sinnerleben und digitale Arbeit betrachten, ist auch die Forschung über Aneignung bzw. Technikaneignung aufschlussreich für das Verständnis des Sinnerlebens, insofern als hier zwar andere Konzepte verwendet werden, sich aber gleichwohl ähnliche Mechanismen beobachten lassen. 


\section{Technikaneignung und Job-Crafting in der digitalisierten Arbeitswelt}

Die Erforschung von Technikaneignung (technology appropriation) richtet den Blick nicht ausschließlich auf den unmittelbaren Umgang mit einem digitalen Gerät oder Tool, sondern vielmehr auf die Dynamiken zwischen den sozialen Praktiken und Materialität. Unter Technikaneignung wird dabei der Prozess verstanden, durch den Technologie übernommen, angepasst und in die eigenen Arbeitspraktiken integriert wird (Janneck 2009; Dourish 2003). Damit akzentuiert Technikaneignung stärker als der Begriff der Techniknutzung die Eigensinnigkeit der Akteur*innen im Umgang mit Technik. Technikaneignung gilt als aktive Bemühung, im Rahmen derer der Nutzen der Technik für das eigene Leben und Arbeiten ausgelotet wird, und durch die auch die (vorgesehene) Techniknutzung verändert werden kann (Janneck 2009). Von Interesse sind vielfach die Widerständigkeiten und der Eigensinn der Akteur*innen, aber auch die Entstehung neuer Praktiken, die eigentlich so nicht vorgesehen sind.

So berichtet Walker (2017) in einer Studie über die Implementierung eines Warenwirtschaftssystems davon, wie Beschäftigte einen Laptop auf einem Einkaufswagen installieren und mit diesem die Regale der Filiale abfahren, um die Realitäten des Computerprogramms mit den Bedingungen vor Ort abzugleichen. In der Studie von Carstensen (2017) wird die Nutzung von Social-collaboration-Plattformen in Unternehmen untersucht, und es wird gezeigt, wie die Beschäftigten die Plattform in unterschiedlicher Weise in ihrer Praktikabilität für die Arbeit bewerten und entsprechend nutzen bzw. nicht nutzen. In Arbeiten über Technikaneignung werden Fragen des Sinnerlebens meist nicht unmittelbar adressiert, ${ }^{9}$ sondern es werden - teilweise mit dem Konzept des Eigensinns - unterschiedliche Arten des Umgangs mit den jeweils neu eingeführten Technologien beschrieben. ${ }^{10}$ Dabei zeigen sich mit dem Interesse an Subjektivität, Eigensinn und Widerständigkeit inhaltlich deutliche Überschneidungen zur Erforschung des Sinnerlebens von Beschäftigten, bei dem es ebenfalls um Formen der (eigensinnigen) Aneignung von Arbeit geht. ${ }^{11}$ Gerade ältere Studien wie die über die Betriebliche Lebenswelt (Volmerg et al. 1986) oder Arbeiten von Regina Becker-Schmidt et al. (1982), in denen die Aneignung der Technik, beispielsweise der Umgang mit der Arbeit am Fließband, als Praxis der Verteidigung von Sinnansprüchen gesehen wurde, sind hier anschlussfähig, denn

\footnotetext{
9 In der Studie von Blazejewski und Walker (2018) werden Formen der Technikaneignung in Verbindung mit Praktiken des Job-Craftings gebracht, womit eine Verbindung zur Meaningful-work-Literatur gelegt ist.

10 In der Studie von Carstensen (2017) wird als ein Problemfeld der eigensinnigen Aneignungspraktiken die wahrgenommene Nutzlosigkeit herausgearbeitet. Die Beschäftigten sehen dann die Plattform als etwas „Schönes“, „Nettes“, was aber bisher noch keine sinnvollen Anwendungsfelder für sie hat. Daran zeigen sich Reflexionen über die Nützlichkeit einzelner Tätigkeiten im Gesamtzusammenhang.

11 Eine hier im Blick zu haltende Differenz bildet der Aneignungsbegriff, der mal stärker auf die Technik konzentriert ist, und an anderer Stelle in einem weiteren Sinne als Aneignung von Arbeit und damit als Praxis der Welterschließung begriffen werden kann. Die Differenz zwischen diesen beiden, einem engeren technikbezogenen und einem weiteren Aneignungsbegriff, wird auch bei Pfeiffer (2004) herausgearbeitet. Pfeiffer kritisiert hier das im Kontext von IuK-Technologien verwendete Aneignungsverständnis, welches häufig ,schlicht als Synonym für ,Umgang mit“" genutzt werde und dem es an philosophischer Einbettung fehle (Pfeiffer 2004, Fn. 56).
} 
auch bei diesen Untersuchungen wurde die Verteidigung von Sinnansprüchen als Praxis der Autonomiewahrung gefasst.

Deutlich wird in den älteren wie auch den aktuelleren Studien der Wunsch, sich Technologien zu eigen zu machen und dabei eigenständige Wege zu finden. Dies kann zu Verweigerung, Nichtnutzung oder auch zu Initiativen der Veränderung der Technologie führen. Die Technologie bestimmt somit nicht die Verhaltensweisen, vielmehr sorgt der Wunsch nach Autonomie dafür, dass eigene Wege der Techniknutzung gefunden werden. Mit Blick auf die Forschungsperspektive finden sich so deutliche Parallelen zu der humanistischen Tradition in der Sinnforschung. Gleichzeitig verweisen die Studien auch auf die organisationalen Rahmenbedingungen und möglichen strukturellen Barrieren des Sinnerlebens. So deutet die Studie von Carstensen (2017) Konsequenzen für das Arbeitserleben der Beschäftigten an und hinterfragt die Rolle der Technik. Wie sich zeigt, ist das Problem vielfach nicht die Technik selbst: „Viel wirkmächtiger als die Digitalisierung scheinen aus Sicht der Subjekte die bestehenden organisatorischen Bedingungen zu sein, in denen sich im Übrigen einige bekannte Problemlagen der Arbeit widerspiegeln: Zeitdruck, Erschöpfung, permanente Reorganisation usw.“ (Carstensen 2017, S. 107). Jenseits von Technik und Materialität zeigt sich so die Wirkmacht organisationaler Machtdynamiken. Deutlich wird damit, dass die digitalen Technologien nicht adäquat in ihrer Wirkung auf Subjekte eingefangen werden können, wenn sie nicht als Element betrieblicher Strategien begriffen werden (Menz et al. 2019). Die gleichsam hinter den Technologien liegenden Dynamiken von Vermarktlichung, veränderten Kontrollstrategien und erhöhtem Arbeitsdruck, die seit vielen Jahren den Wandel von Arbeit charakterisieren, prägen somit auch substanziell die Arbeitswirklichkeiten mit digitalen Technologien.

\section{Fazit und Ausblick: Sinn und Aneignung in der digitalen Arbeitswelt}

Wie der Durchgang durch die verschiedenen Zweige der Digitalisierungsdiskussion zeigt, überwiegen Vermutungen und möglicherweise auch Hoffnungen die tatsächlichen Kenntnisse über den Sinn der Arbeit und das Sinnerleben in der Arbeit in Zeiten der Digitalisierung. Ein Kernproblem sämtlicher Diskussionen bleibt die Unschärfe des Sinnbegriffs, welcher sowohl auf die gesellschaftliche Bedeutung der Arbeit wie auch auf die konkreten Erfahrungen des Sinnerlebens von Beschäftigten bezogen wird. Die zur Differenzierung der unterschiedlichen Stränge eingeführte Heuristik ermöglicht, den Status der jeweiligen Diskurse differenziert betrachten zu können. Die Durchsicht der verschiedenen Diskurse zeigte unterschiedliche Vertiefungsgrade der Diskursstränge. Weiterhin wird deutlich, dass die Digitalisierung der Arbeitswelt die Sinnfrage in mehrfacher Hinsicht neu stellt. Dabei wird vielfach an alte Diskussionen über das „Ende der Arbeit“ angeschlossen und es treten ähnliche Muster des Debattenverlaufes wie in früheren Diskursen über Technik und Arbeit auf. Mit Blick auf die individuelle Bedeutung von Arbeit und die Erfahrung von Sinn in der Arbeit scheint die Digitalisierung zu einer Verschärfung bereits bestehender Problemlagen beizutragen, da sie belastende Tendenzen der Arbeitswelt wie Zeitdruck und Arbeitsverdichtung verstärkt. 
Die wenigen Studien, die das Sinnerleben und dessen Barrieren unmittelbar untersuchen, deuten darauf hin, dass sich die Nutzung digitaler Technologien in ihrer Wirkung auf das Sinnerleben in der Arbeit insbesondere über die wahrgenommene Autonomie begreifen lässt: Je stärker die Techniknutzung einen Autonomiegewinn impliziert, desto positiver ist sie für das Sinnerleben, je restriktiver sie ist, desto eher lässt sich die Technik als Barriere für ein positives Sinnerleben begreifen (Stein et al. 2018). Solche Befunde sind äußerst anschlussfähig an die Erforschung des Sinnerlebens, insofern sie die Zentralstellung der Autonomiewahrnehmung bzw. der Verteidigung von Handlungsspielräumen bei der Erfahrung von Sinnerfüllung bestätigen. Auch Studien über Technikaneignung, die das Sinnerleben nicht unmittelbar untersuchen, akzentuieren die Maßnahmen der Beschäftigten, Autonomie zu wahren bzw. zurückzugewinnen (Walker 2017; Carstensen 2017). Weiterhin zeigte sich, dass die Einführung digitaler Technologien nicht adäquat verstanden werden kann, wenn man ihre Einbettung in organisationale Gefüge und Strategien der Leistungssteuerung nicht berücksichtigt. Viele der neuen Belastungen, die im Kontext der Digitalisierungsdiskussion angesprochen werden, lassen sich als Folge von Vermarktlichungstendenzen begreifen, durch welche grundlegende Veränderungen der Arbeitsorganisation angestoßen wurden.

Deutlich werden zudem verschiedene Forschungsdesiterata, an die im Rahmen weiterer Forschung angeschlossen werden kann: So deuten die Untersuchungen über den Sinn in der Arbeit mögliche Differenzierungen der Aneignungsprozesse von Arbeit auf unterschiedlichen Qualifikationsniveaus in digitalen Kontexten an, die zum Gegenstand genauerer Analysen werden können. Beispielsweise spielt in verschiedenen Tätigkeitsfeldern Autonomie eine zentrale Rolle; gleichwohl sind besonders Hochqualifizierte von der „Herrschaft durch Autonomie“ (Moldaschl 2001) und damit von neuen Steuerungsformen betroffen, die zwar neue Autonomiespielräume gewähren, aber über den entstehenden Leistungsdruck das Sinnerleben gefährden können. ${ }^{12}$

Wenn sich aktuell abzeichnet, dass viele der subjektiv erlebten Konsequenzen der Digitalisierung von Arbeitsprozessen sich letztlich auf veränderte Handlungsspielräume, die Beschleunigung der Arbeitsprozesse, die Zunahme von Zeit- und Leistungsdruck und damit bekannte Muster der Veränderung von Arbeit herunterbrechen lassen, bleibt damit ein wichtiger Aspekt der Erfahrung des Digitalen weitgehend unberücksichtigt. Noch ist wenig darüber bekannt, inwieweit die Virtualisierung von Arbeitsprozessen sowie der Arbeitsumgebung das Arbeitserleben verändert, und zwar nicht nur mit Blick auf Autonomie, Kommunikation und die kompensatorischen Leistungen der Subjekte zur Aufrechterhaltung der Arbeitsprozesse, sondern mit Blick auf die Möglichkeit der Beschäftigten, sich mit der Tätigkeit und darüber hinaus mit der Welt in Beziehung zu setzen (Rosa 2016). Wenn Informationen über Arbeitsprozesse nur noch als virtuelle Abbildung zugänglich sind, Meetings in digitalen Videokonferenzräumen stattfinden und die unmittelbare sinnliche Erfahrung über verschiedene Wahrnehmungskanäle ausbleibt, ist davon auszugehen, dass die-

\footnotetext{
12 Im Projekt: Digitale Entfremdung und Aneignung von Arbeit: Entfremdungserfahrungen in digitaler Dienstleistungsarbeit sollen daher Dynamiken von Entfremdung und Aneignung bei Beschäftigten verschiedener Qualifikationsniveaus empirisch zugänglich gemacht werden (Hardering und Nachtwey 2018).
} 
se fehlende Erfahrungsdichte Folgen für das Sinnerleben der Beschäftigten haben kann. Wie sich diese Abstraktifizierung und Entsinnlichung von Arbeit ${ }^{13}$ auf die Erfahrungswelten der Beschäftigten und ihre Weltaneignung genau auswirkt, und wie sich diese Erfahrungen in den Erzählungen der Beschäftigten und ihren Emotionen abbilden, ist somit ein wichtiger Gegenstand zukünftiger Arbeitsforschung.

Funding Open Access funding enabled and organized by Projekt DEAL.

Open Access Dieser Artikel wird unter der Creative Commons Namensnennung 4.0 International Lizenz veröffentlicht, welche die Nutzung, Vervielfältigung, Bearbeitung, Verbreitung und Wiedergabe in jeglichem Medium und Format erlaubt, sofern Sie den/die ursprünglichen Autor(en) und die Quelle ordnungsgemäß nennen, einen Link zur Creative Commons Lizenz beifügen und angeben, ob Änderungen vorgenommen wurden.

Die in diesem Artikel enthaltenen Bilder und sonstiges Drittmaterial unterliegen ebenfalls der genannten Creative Commons Lizenz, sofern sich aus der Abbildungslegende nichts anderes ergibt. Sofern das betreffende Material nicht unter der genannten Creative Commons Lizenz steht und die betreffende Handlung nicht nach gesetzlichen Vorschriften erlaubt ist, ist für die oben aufgeführten Weiterverwendungen des Materials die Einwilligung des jeweiligen Rechteinhabers einzuholen.

Weitere Details zur Lizenz entnehmen Sie bitte der Lizenzinformation auf http://creativecommons.org/ licenses/by/4.0/deed.de.

\section{Literatur}

Badura, Bernhard. 2018. Über sinnstiftende Arbeit: Editorial. In Fehlzeiten-Report 2018: Sinn erleben Arbeit und Gesundheit, Hrsg. Bernhard Badura, Antje Ducki, Helmut Schröder, Joachim Klose, und Markus Meyer, 1-7. Berlin: Springer.

Badura, Bernhard, Antje Ducki, Helmut Schröder, Joachim Klose, und Markus Meyer (Hrsg.). 2018. Fehlzeiten-Report 2018: Sinn erleben - Arbeit und Gesundheit. Berlin: Springer.

Bailey, Catherine, Adrian Madden, Kerstin Alfes, Amanda Shantz, und Emma Soane. 2017. The mismanaged soul. Existential labor and the erosion of meaningful work. Human Resource Management Review 27(3):416-430. https://doi.org/10.1016/j.hrmr.2016.11.001.

Bailey, Catherine, Ruth Yeoman, Adrian Madden, Marc Thompson, und Gary Kerridge. 2018. A review of the empirical literature on meaningful work: progress and research agenda. Human Resource Development Review 9:83-113. https://doi.org/10.1177/1534484318804653.

Becker-Schmidt, Regina, Uta Brandes-Erloff, Marva Karrer, G. Axeli Knapp, Mechthild Rumpf, und Beate Schmidt. 1982. Nicht wir haben die Minuten, die Minuten haben uns: Zeitprobleme und Zeiterfahrungen von Arbeitermüttern in Fabrik und Familie: Studie zum Projekt „Probleme lohnabhängig arbeitender Mütter". Bonn: Neue Gesellschaft.

Blazejewski, Susanne, und Eva-Maria Walker. 2018. Digitalization in retail work: coping with stress through job crafting. Management Revue 29(1):79-100. https://doi.org/10.5771/0935-9915-2018-179.

Bonin, Holger, Terry Gregory, und Ulrich Zierahn. 2015. Übertragung der Studie von Frey/Osborne (2013) auf Deutschland. Mannheim: Zentrum für Europäische Wirtschaftsforschung (ZEW) (ZEW Kurzexpertise, 57). https://www.econstor.eu/bitstream/10419/123310/1/82873271X.pdf. Zugegriffen: 16. Okt. 2020.

Bregman, Rutger. 2019. Utopien für Realisten. Die Zeit ist reif für die 15-Stunden-Woche, offene Grenzen und das bedingungslose Grundeinkommen. Hamburg: Rowohlt.

Briken, Kendra, Shiona Chillas, Martin Krzywdzinski, und Abigail Marks. 2017. The new digital workplace: how new technologies revolutionise work. London: Macmillan Education Palgrave.

13 Die Rede von der Abstraktifizierung und der Entsinnlichung von Arbeit findet sich insbesondere im Kontext des Diskurses über die Informatisierung von Arbeit (Schmiede 1996). 
Broy, Manfred, und Richard David Precht. 2017. Daten essen Seele auf. Die Zeit 5/2017. https://www.zeit. de/2017/05/digitalisierung-revolution-technik-seele-menschen-grundrechte/seite-2. Zugegriffen: 16. Okt. 2020.

Bundesministerium für Arbeit und Soziales (BMAS). 2016. Monitor Digitalisierung am Arbeitsplatz. Aktuelle Ergebnisse einer Betriebs- und Beschäftigtenbefragung. Berlin: BMAS.

Carstensen, Tanja. 2015. Neue Anforderungen und Belastungen durch digitale und mobile Technologien. WSI-Mitteilungen 3:187-193.

Carstensen, Tanja. 2017. Digitalisierung als eigensinnige soziale Praxis. Arbeit 26(1):87-110. https://doi. org/10.1515/arbeit-2017-0005.

DGB-Index Gute Arbeit. 2017. Verbreitung, Folgen und Gestaltungsaspekte der Digitalisierung in der Arbeitswelt. Auswertungsbericht auf Basis des DGB-Index Gute Arbeit 2016.

Dornes, Martin. 2016. Macht der Kapitalismus depressiv? Frankfurt a. Main: Fischer.

Dourish, Paul. 2003. The appropriation of interactive technologies: some lessons from placeless documents. Computer Supported Cooperative Work (CSCW) 12(4):465-490. https://doi.org/10.1023/A: 1026149119426.

Eisenmann, Martin, und Tobias Wienzek. 2018. Stellt Digitalisierung eine Gefahr oder eine Chance für das Sinnerleben der Arbeit dar? In Fehlzeiten-Report 2018: Sinn erleben - Arbeit und Gesundheit; Zahlen, Daten, Analysen aus allen Branchen der Wirtschaft, Hrsg. Bernhard Badura, Antje Ducki, Helmut Schröder, Joachim Klose, und Markus Meyer, 177-188. Berlin: Springer.

Frey, Carl Benedikt, und Michael A. Osborne. 2013. The future of employment: how susceptible are jobs to computerization? Oxford. https://www.oxfordmartin.ox.ac.uk/downloads/academic/The_Future_of_ Employment.pdf. Zugegriffen: 9. Nov. 2020.

Gimpel, Henner, Julia Lanzl, Tobias Manner-Romberg, und Niclas Nüske. 2018. Digitaler Stress in Deutschland. Eine Befragung von Erwerbstätigen zu Belastung und Beanspruchung durch Arbeit mit digitalen Technologien. Working Paper der Hans-Böckler-Stiftung, Nummer 101.

Graham, Mark, Isis Hjorth, und Vili Lehdonvirta. 2017. Digital labour and development. Impacts of global digital labour platforms and the gig economy on worker livelihoods. Transfer 23(2):135-162. https:// doi.org/10.1177/1024258916687250.

Hackman, J. Richard, und Greg R. Oldham. 1980. Work redesign. Reading: Addison-Wesley.

Hardering, Friedericke. 2015. Meaningful work: Sinnvolle Arbeit zwischen Subjektivität, Arbeitsgestaltung und gesellschaftlichem Nutzen. Österreichische Zeitschrift für Soziologie 40(4):391-410. https://doi.org/10.1007/s11614-015-0181-7.

Hardering, Friedericke. 2017a. Die Suche nach dem Sinn: Zur Zukunft der Arbeit. Aus Politik und Zeitgeschichte 26:4-10.

Hardering, Friedericke. 2017b. Wann erleben Beschäftigte ihre Arbeit als sinnvoll? Befunde aus einer Untersuchung über professionelle Dienstleistungsarbeit. Zeitschrift für Soziologie 46(1):39-54. https:// doi.org/10.1515/zfsoz-2017-1003.

Hardering, Friedericke. 2018. Die Sinnsuche der Generation Y. Zum Wandel von Ansprüchen an den Sinn (in) der Arbeit. In Fehlzeiten-Report 2018: Sinn erleben - Arbeit und Gesundheit, Hrsg. Bernhard Badura, Antje Ducki, Helmut Schröder, Joachim Klose, und Markus Meyer, 75-83. Berlin: Springer.

Hardering, Friedericke. 2020. Sinn in der Arbeit: Überblick über Grundbegriffe und aktuelle Debatten. Wiesbaden: Springer.

Hardering, Friedericke, und Oliver Nachtwey. 2018. Digitale Entfremdung und Aneignung von Arbeit. Unveröffentlichter Antrag an DFG und SNF.

Heßler, Martina. 2016. Zur Persistenz der Argumente im Automatisierungsdiskurs. Aus Politik und Zeitgeschichte 66(18-19):17-24.

Hirsch-Kreinsen, Hartmut. 2018. Einleitung: Digitalisierung industrieller Arbeit. In Digitalisierung industrieller Arbeit. Die Vision Industrie 4.0 und ihre sozialen Herausforderungen, Hrsg. Hartmut HirschKreinsen, Peter Ittermann, und Jonathan Niehaus, 10-31. Baden-Baden: edition sigma.

Hirsch-Kreinsen, Hartmut, Peter Ittermann, und Jonathan Niehaus (Hrsg.). 2018. Digitalisierung industrieller Arbeit. Die Vision Industrie 4.0 und ihre sozialen Herausforderungen. Baden-Baden: edition sigma.

Hoose, Fabian. 2018. Digitale Arbeit. Strukturen eines Forschungsfeldes. Duisburg Essen (IAQ-Forschung, 2018/03). http://www.iaq.uni-due.de/iaq-forschung/2018/fo2018-03.pdf. Zugegriffen: 16. Okt. 2020.

Isaksen, Jesper. 2000. Constructing meaning despite the drudgery of repetitive work. Journal of Humanistic Psychology 40(3):84-107. https://doi.org/10.1177/0022167800403008.

Janneck, Monique. 2009. Recontextualising technology in appropriation processes. In Handbook of research on socio-technical design and social networking systems, Hrsg. Brian Whitworth, Aldo de Moor, 153-166. Hershey: Information Science Reference. 
Jürgens, Kerstin. 2014. „Sinnvolle Arbeit“ - der Maßstab „Guter Arbeit“. In Arbeit in Europa: Marktfundamentalismus als Zerreißprobe, Hrsg. Klaus Dörre, Kerstin Jürgens, und Ingo Matuschek, 325-338. Frankfurt a. Main: Campus.

Jürgens, Kerstin, Reiner Hoffmann, und Christina Schildmann. 2017. Arbeit transformieren! Denkanstöße der Kommission „Arbeit der Zukunft“. Bielefeld: transcript.

Kämpf, Tobias. 2015. „Ausgebrannte Arbeitswelt“ - Wie erleben Beschäftigte neue Formen von Belastung in modernen Feldern der Wissensarbeit? Berliner Journal für Soziologie 25(1-2):133-159. https:// doi.org/10.1007/s11609-015-0278-7.

Kost, Dominique, Christian Fieseler, und Sut I. Wong. 2018. Finding meaning in a hopeless place? The construction of meaningfulness in digital microwork. Computers in Human Behavior 82:101-110. https://doi.org/10.1016/j.chb.2018.01.002.

Lips-Wiersma, Marjolein. 2001. The influence of spiritual "meaning-making" on career behavior. Journal of Management Development 21(7):497-520.

Lips-Wiersma, Marjolein, Sarah Wright, und Bryan Dik. 2016. Meaningful work: differences among blue-, pink-, and white-collar occupations. Career Development International 21(5):534-551. https://doi. org/10.1108/CDI-04-2016-0052.

Littig, Beate, und Markus Spitzer. 2011. Arbeit neu. Erweiterte Arbeitskonzepte im Vergleich. Literaturstudie zum Stand der Debatte um erweiterte Arbeitskonzepte. Arbeitspapier 229. Düsseldorf: HansBöckler-Stiftung.

Mason, Paul. 2016. Postkapitalismus: Grundrisse einer kommenden Ökonomie. Berlin: Suhrkamp.

Matthes, Joachim (Hrsg.). 1983. Krise der Arbeitsgesellschaft? Verhandlungen des 21. Deutschen Soziologentages in Bamberg 1982. Frankfurt a. Main: Campus.

Menz, Wolfgang, Sarah Nies, und Dieter Sauer. 2019. Digitale Kontrolle und Vermarktlichung. PROKLA 49(195):181-200. https://doi.org/10.32387/prokla.v49i195.1808.

Moldaschl, Manfred. 2001. Herrschaft durch Autonomie - Dezentralisierung und widersprüchliche Arbeitsanforderungen. In Entwicklungsperspektiven von Arbeit, Hrsg. B. Lutz, 132-164. Berlin: de Gruyter.

Müller-Thur, Kathrin, Peter Angerer, Ulrike Körner, und Nico Dragano. 2018. Arbeit mit digitalen Technologien, psychosoziale Belastungen und potenzielle gesundheitliche Konsequenzen: Wo gibt es $\mathrm{Zu}-$ sammenhänge? ASU Arbeitsmedizin Sozialmedizin Umweltmedizin 52:388-391.

Nachtwey, Oliver, und Philipp Staab. 2015. Die Avantgarde des digitalen Kapitalismus. Mittelweg 36 24(6):59-84.

Neckel, Sighard, und Greta Wagner (Hrsg.). 2013. Leistung und Erschöpfung: Burnout in der Wettbewerbsgesellschaft. Frankfurt a. Main: Suhrkamp.

Nierling, Linda, und Bettina-Johanna Krings. 2019. Digitalisierung und erweiterte Arbeit. In Tätigsein in der Postwachstumsgesellschaft, Hrsg. Irmi Seidl, Angelika Zahrnt, 175-190. Marburg: Metropolis Verlag.

Nies, Sarah. 2015. Nützlichkeit und Nutzung von Arbeit. Beschäftigte im Konflikt zwischen Unternehmenszielen und eigenen Ansprüchen. Baden-Baden: edition sigma.

Ohlbrecht, Heike. 2018. Arbeitswelt und Gesundheit: Ein gesundheitssoziologischer Blick auf die Herausforderungen der Arbeitswelt 4.0. In Medizinische Soziologie trifft Medizinische Pädagogik, Hrsg. Heike Ohlbrecht, Astrid Seltrecht, 117-137. Wiesbaden: Springer.

Orlikowski, Wanda J. 2016. Digital work: a research agenda. In A research agenda for management and organization studies, Hrsg. Barbara Czarniawska-Joerges, 88-95. Cheltenham: Edward Elgar Publishing.

Pfeiffer, Sabine. 2004. Arbeitsvermögen: Ein Schlüssel zur Analyse (reflexiver) Informatisierung. Wiesbaden: VS.

Pfeiffer, Sabine. 2015. Industrie 4.0 und die Digitalisierung der Produktion - Hype oder Megatrend? Aus Politik und Zeitgeschichte 65(31/32):6-12.

Pongratz, Hans J., und Sarah Bormann. 2017. Online-Arbeit auf Internet-Plattformen. Empirische Befunde zum ,Crowdworking' in Deutschland. AIS Studien, Bd. 10, 158-181.

Precht, Richard David. 2018. Jäger, Hirten, Kritiker: Eine Utopie für die digitale Gesellschaft. München: Goldmann.

Rifkin, Jeremy. 1995. Das Ende der Arbeit und ihre Zukunft: Neue Konzepte für das 21. Jahrhundert. Frankfurt a. Main: Fischer.

Rosa, Hartmut. 2011. Entfremdung in der Spätmoderne. Umrisse einer kritischen Theorie der sozialen Beschleunigung. In Nachrichten aus den Innenwelten des Kapitalismus, Hrsg. Cornelia Koppetsch, 221-252. Wiesbaden: VS Verlag fur Sozialwissenschaften. 
Rosa, Hartmut. 2012. Arbeit und Entfremdung. In Kapitalismustheorie und Arbeit: Neue Ansätze soziologischer Kritik, Hrsg. Klaus Dörre, Dieter Sauer, und Volker Wittke, 410-420. Frankfurt a. Main: Campus.

Rosa, Hartmut. 2016. Resonanz: Eine Soziologie der Weltbeziehung, 2. Aufl., Berlin: Suhrkamp.

Rosso, Brent D., Kathryn H. Dekas, und Amy Wrzesniewski. 2010. On the meaning of work: a theoretical integration and review. Research in Organizational Behavior 30:91-127. https://doi.org/10.1016/j. riob.2010.09.001.

Rosswog, Tobi. 2018. After Work: Radikale Ideen für eine Gesellschaft jenseits der Arbeit. München: oekom.

Schmiede, Rudi (Hrsg.). 1996. Virtuelle Arbeitswelten. Arbeit, Produktion und Subjekt in der „Informationsgesellschaft“. Berlin: edition sigma.

Schnell, Tatjana, Thomas Höge, und Edith Pollet. 2013. Predicting meaning in work: Theory, data, implications. The Journal of Positive Psychology 8(6):543-554. https://doi.org/10.1080/17439760.2013. 830763.

Schwemmle, Michael, und Peter Wedde. 2012. Digitale Arbeit in Deutschland. Potenziale und Problemlagen. Report, Medienpolitik. Bonn: Friedrich-Ebert-Stiftung.

Srnicek, Nick, und Alex Williams. 2016. Die Zukunft erfinden: Postkapitalismus und eine Welt ohne Arbeit. Berlin: edition TIAMAT.

Staab, Philipp, und Oliver Nachtwey. 2016. Die Digitalisierung der Dienstleistungsarbeit. Aus Politik und Zeitgeschichte 18-19:24-31.

Stein, Mari-Klara, Erica L. Wagner, Pamela Tierney, Sue Newell, und Robert D. Galliers. 2018. Datification and the Pursuit of Meaningfulness in Work. Journal of Management Studies 29:29. https://doi. org/10.1111/joms.12409.

Symon, Gillian, und Rebecca Whiting. 2019. The sociomaterial negotiation of social entrepreneurs' meaningful work. Journal of Management Studies 56(3):655-684. https://doi.org/10.1111/joms.12421.

Vašek, Thomas. 2017. Im Arbeitskreis der Algorithmen. Hohe Luft Business Class, 7. März. https://www. hoheluft-businessclass.com/im-arbeitskreis-der-algorithmen. Zugegriffen: 6. Nov. 2020.

Vobruba, Georg. 2019. Entkoppelung von Arbeit und Einkommen: Das Grundeinkommen in der Arbeitsgesellschaft, 3. Aufl., Wiesbaden: Springer.

Volmerg, Brigitte, Eva Senghaas-Knobloch, und Thomas Leithäuser (Hrsg.). 1986. Betriebliche Lebenswelt: Eine Sozialpsychologie Industrieller Arbeitsverhältnisse. Opladen: Westdeutscher Verlag.

Voswinkel, Stephan. 2015. Sinnvolle Arbeit leisten - Arbeit sinnvoll leisten. Arbeit: Zeitschrift für Arbeitsforschung, Arbeitsgestaltung und Arbeitspolitik 24(1-2):31-48.

Walker, Eva-Maria. 2017. Subjektive Aneignungspraktiken digitaler Technologien und die zugrundeliegenden Gerechtigkeitsansprüche der Beschäftigten. Arbeit https://doi.org/10.1515/arbeit-2017-0021.

Welskop-Deffaa, Eva M. 2018. Erwerbsverläufe digitaler Nomaden. In Hybride Erwerbsformen, Hrsg. Andrea D. Bührmann, Uwe Fachinger, und Eva M. Welskop-Deffaa, 107-129. Wiesbaden: Springer.

Wendsche, Johannes, und Andrea Lohmann-Haislah. 2017. Detachment als Bindeglied zwischen psychischen Arbeitsanforderungen und ermüdungsrelevanten psychischen Beanspruchungsfolgen: Eine Metaanalyse. Zeitschrift für Arbeitswissenschaft 71(1):52-70. https://doi.org/10.1007/s41449-0170044-0.

Friedericke Hardering Dr. phil., geb. 1980, ist Professorin für Sozialen Wandel in der digitalisierten Gesellschaft an der FH Münster. Aktuell leitet sie in Kooperation mit Oliver Nachtwey das DFG/SNF-Projekt „Digitale Entfremdung und Aneignung von Arbeit“. Arbeitsschwerpunkte: Zukunft der Arbeit, Sinn und Zufriedenheit in der Arbeitswelt, Digitalisierung. Publikationen: Sinn in der Arbeit (2020) Wiesbaden: Springer VS (essentials); zusammen mit Mascha Will-Zocholl. 2019. Zwischen Sinngestaltung und Sinnbewahrung - Aneignungsweisen in hochqualifizierter Dienstleistungsarbeit Berliner Journal für Soziologie, 29, 273-298. 